\title{
Determinants of female fecundity in a simultaneous hermaphrodite: the role of polyandry and food availability
}

\author{
Tim Janicke $\cdot$ Peter Sandner $・$ Lukas Schärer
}

Received: 5 February 2010/ Accepted: 17 June 2010

(C) Springer Science+Business Media B.V. 2010

\begin{abstract}
Classical sexual selection theory assumes that the reproductive success of females is primarily limited by the resources available for egg production rather than by the number of mating partners. However, there is now accumulating evidence that multiple mating can entail fitness costs or benefits for females. In this study we investigated the effect of polyandry (i.e., the mating with different mating partners) and food availability on the reproductive output of the female sex function in an outcrossing simultaneous hermaphrodite, the free-living flatworm Macrostomum lignano. We exposed virgin worms to different group sizes, a treatment that has previously been shown to affect the level of polyandry in this species. Moreover, we manipulated the food availability throughout the subsequent egg laying period, during which the worms were kept in isolation. The number of offspring produced was used as an estimate of female fecundity. We found that food availability, but not group size, had a significant effect on female fecundity. Additionally, female fecundity was positively correlated with the number of stored sperm in the female sperm-storage organ at the time of isolation, but it was not correlated with body or ovary size of the worms. Our results suggest that female fecundity in M. lignano is primarily determined by the resources available for egg production, and not by the level of polyandry, confirming classic sexual selection theory for simultaneous hermaphrodites.
\end{abstract}

Keywords Body size · Food availability - Group size · Macrostomum lignano · Multiple mating $\cdot$ Polyandry $\cdot$ Sperm storage

\section{Introduction}

Classical sexual selection theory assumes that the reproductive output of females is primarily limited by the resources available for egg production rather than by the number of mating partners (Bateman 1948). Consequently, females are expected to copulate only once or a few times to obtain sufficient sperm to fertilize their eggs, especially if mating

T. Janicke $(\bowtie) \cdot$ P. Sandner $\cdot$ L. Schärer

Evolutionary Biology, Zoological Institute, University of Basel, Vesalgasse 1, 4051 Basel, Switzerland e-mail: tim.janicke@gmx.de 
entails costs, such as time and energy expenses (Daly 1978), a higher predation risk (Rowe 1994), an increased exposure to parasites and infections (Thrall et al. 1997), a reduced immune function (Rolff and Siva-Jothy 2002), physical injuries (Crudgington and SivaJothy 2000) or a reduced lifespan caused by male accessory gland products (Chapman et al. 1995; Green and Tregenza 2009; but see Priest et al. 2008; Reinhardt et al. 2009). However, over the last decades empirical studies have revealed that multiple mating in females is widespread across many animal taxa. There is now accumulating evidence that multiple mating in females is not only driven by male promiscuity, but may in fact represent an adaptive behavioural strategy that increases female fitness and often outweighs the costs of mating (for reviews see Arnqvist and Nilsson 2000; Jennions and Petrie 2000; Hosken and Stockley 2003; Andersson 2005; Simmons 2005).

Hypotheses for the evolution and maintenance of multiple mating in females either rely on material (direct) or genetic (indirect) benefits (Reynolds 1996). Material benefits refer to fitness gains mediated by resources that are provided by males and can therefore be obtained both from multiple mating with different males (hereafter called polyandry) or with the same male (hereafter called repeated matings), as long as males do not become depleted in the commodity that is beneficial to females. In contrast, genetic benefits are related to genetic diversity among male gametes, which can only be augmented considerably by polyandry (but see Yasui 1997 for an argument that considers genetic diversity within ejaculates). To avoid confusion we here use 'multiple mating' as a general term for individuals that mate more than once, irrespective of whether this involves 'repeated matings' or 'polyandry'.

Material benefits of multiple mating encompass resources that females obtain from males, such as nutrients (e.g., prey items, seminal products), parental investment and/or protection against conspecifics or predators (e.g., Gwynne 1984; Vahed 1998; Engqvist 2007; reviewed in Reynolds 1996; Arnqvist and Nilsson 2000). Furthermore, the receipt of sperm can represent a material benefit, if female reproductive success is limited by the number of sperm that is available to fertilize all the eggs (e.g., Pitnick 1993; Levitan and Petersen 1995; Fjerdingstad and Boomsma 1998; Diaz et al. 2010). Several empirical studies across a wide range of taxa have demonstrated that repeated matings are advantageous to females (e.g., Wagner et al. 2001; Fedorka and Mousseau 2002; Schwartz and Peterson 2006; Klemme et al. 2007), which suggests that direct benefits may promote the evolution of multiple mating. Likewise, a meta-analysis of 122 studies focussing on female fitness consequences of multiple mating indicated that direct benefits alone can explain the evolutionary maintenance of multiple mating in insects (Arnqvist and Nilsson 2000).

Genetic benefits derived from polyandrous mating can also be manifold (for reviews on genetic benefits see Yasui 1998; Jennions and Petrie 2000; Zeh and Zeh 2001; Simmons 2005). For instance, females might benefit from polyandry by means of a bet-hedging strategy that lowers the probability of mating only with inferior males (in case of errorprone mate choice abilities) or with males that carry genes that are maladapted to future environments (in case of unpredictable environments) (Watson 1991). Furthermore, it has been argued that females are polyandrous in order to minimize the risk and the associated fitness cost of fertilization by genetically incompatible sperm (Zeh and Zeh 1996, 1997). Contrary to the other genetic benefit models the 'genetic incompatibility hypothesis' assumes that the interaction between maternal and paternal haplotypes involves fitness consequences that are non-additive. Given that genetic compatibility is expected to be required for a normal embryogenesis, the 'genetic compatibility hypothesis' not only predicts a positive effect of polyandry on the quality of the offspring, but also on the number of viable offspring produced (Zeh and Zeh 1996). 
Empirical studies indicate that there is no consistency in the effect of polyandry on female fecundity across taxa. For instance, mating with multiple males has been shown to be beneficial to females in echinoderms (Evans and Marshall 2005), insects (Tregenza and Wedell 1998; Fedorka and Mousseau 2002; Dunn et al. 2005), fishes (Evans and Magurran 2000) and reptiles (LaDage et al. 2008), but it can also be associated with a reduction of the female's reproductive output, as reported for many insect species (e.g., Orsetti and Rutowski 2003; Bybee et al. 2005; Ronkainen et al. 2010). At the same time, several studies found no effect of polyandry on female fecundity in insects (e.g., Baker et al. 2001; Schwartz and Peterson 2006; House et al. 2009).

Compared to this large body of empirical studies on separate-sexed organisms, very little attention has been placed on the fitness consequences of polyandry in simultaneous hermaphrodites, i.e. organisms that produce sperm and eggs at the same time. In his seminal paper on sexual selection in simultaneous hermaphrodites, Charnov (1979) assumed that Bateman's principle (Bateman 1948) is also valid for simultaneously hermaphroditic animals. However, his line of reasoning that "fertilized egg production by an individual is limited not by the ability to get sperm, but by resources allocated to eggs" (Charnov 1979) differs slightly from Bateman's principle, because it only refers to an effect of sperm availability on female fecundity, which is not necessarily related to the number of mating partners (unless sperm donors get sperm depleted). Therefore, Charnov (1979) primarily made a prediction for the effect of repeated matings, but not for the effect of polyandry on female fecundity. Charnov (1979) did not clarify if this difference was in any way intentional or just the result of a slightly different phrasing. Either way, empirical tests of the validity of both Bateman's principle and Charnov's hypothesis are still scarce in simultaneous hermaphrodites (but see e.g., Marshall and Evans 2007; Sprenger et al. 2008).

Studying the costs and benefits of multiple mating in simultaneous hermaphrodites reveals several interesting differences to separate-sexed organisms. First, theoretical analyses suggest that matings in simultaneous hermaphrodites may often be more harmful to the female function than expected for separate-sexed animals (Michiels and Koene 2006). This is because in contrast to females, simultaneous hermaphrodites should remate even if mating entails severe fitness costs for the female function, as long as they can compensate these costs by a sufficiently high male fitness benefit (Michiels and Koene 2006). Second, in many simultaneously hermaphroditic species mating occurs reciprocally (i.e., each partner both donates and receives sperm during each mating), which inevitably links the mating strategies of both sex functions (Michiels 1998). Given that multiple mating might be beneficial for one sex function, but costly for the other function, a tradeoff between the optimal male and female mating strategies might arise within one individual, i.e. being eager to mate in one sex function versus being reluctant in the other sex function (Bedhomme et al. 2009; Janicke and Schärer 2009b). Third, empirical data on female fitness consequences of multiple mating are crucial to resolve a longstanding debate on the preferred mating role in simultaneous hermaphrodites. Here, the preferred mating role normally refers to the sex function that on average yields a higher expected benefit from an additional mating. Contrary to Charnov's (1979) prediction that simultaneous hermaphrodites "copulate not so much to gain sperm to fertilize their eggs as to give sperm away", it has also been hypothesised that hermaphrodites mate preferentially in the female sex function (Leonard 2005, 2006). To date, empirical tests of these hypotheses have primarily aimed to demonstrate whether individuals trade male or female gametes, in order to infer indirectly in which sex role they mate preferentially. On the one hand, there are studies indicating that simultaneous hermaphrodites trade eggs during mating, which 
suggests an overall preference to donate sperm to fertilize the partner's eggs, i.e. to mate in the male role (e.g., Fischer 1980; Sella 1985). But on the other hand, there are also studies that provide evidence for sperm trading, which may suggest that they copulate primarily in order to receive sperm, i.e. to mate in the female role (e.g., Leonard and Lukowiak 1991; Vreys and Michiels 1998; Anthes et al. 2005).

In order to shed light on the importance of Bateman's principle for simultaneous hermaphrodites, we studied the effect of polyandry and food availability on female fecundity in the outcrossing simultaneously hermaphroditic flatworm Macrostomum lignano. Copulations in this species are reciprocal (Schärer et al. 2004) and it has been shown that worms are highly promiscuous when they are exposed to multiple potential mating partners (Janicke and Schärer 2009a). Furthermore, worms that allocate relatively more resources towards the male sex function mate more frequently and it has been argued that multiple mating in this species may primarily be driven by the male sex function (Janicke and Schärer 2009b). However, whether multiple mating causes either benefits or costs for the female sex function is currently unknown. We manipulated the group size and the food availability of worms to infer how both factors affect female fecundity (measured as the number of offspring produced). Furthermore, we studied how morphological traits of the worms and the amount of received sperm in storage relate to female fecundity.

\section{Methods}

Study organism

The free-living flatworm Macrostomum lignano (Macrostomorpha, Platyhelminthes) is an outcrossing simultaneous hermaphrodite of the intertidal meiofauna of the Northern Adriatic Sea (Ladurner et al. 2005). In mass cultures worms are maintained at $20^{\circ} \mathrm{C}$ in glass Petri dishes filled with f/2 medium (Andersen et al. 2005) and fed with the algae Nitzschia curvilineata. Under these conditions, body length of a fully grown worm reaches on average $1.5 \mathrm{~mm}$ and the generation time is about 18 days. The worms are transparent allowing non-invasive measurement of internal morphological traits, such as testis size, ovary size and the size of the seminal vesicle (which stores the produced sperm before it is transferred to the mating partners). The transparency also makes it possible to count the sperm that is stored in the female sperm-storage organ (hereafter called 'antrum', plural 'antra') in vivo. The antrum contains the fertilized egg until it is released through the ciliated vagina (Ladurner et al. 2005; Vizoso et al. 2010).

\section{Experimental design}

To test for an effect of polyandry and food availability on female fecundity we used a fully factorial design, in which we manipulated both the group size and the food level. On day 1 we pooled 1,200 adult worms from mass cultures and distributed them equally to 6 Petri-dishes filled with $\mathrm{f} / 2$ medium and a dense algae layer and allowed them to lay eggs. On day 3 we removed all adults from the Petri-dishes, which assured that their offspring did not differ by more than 2 days in age. On day 8 we pooled all hatchlings from the 6 Petri-dishes and isolated 840 individuals in wells of 24 well-plates, which were filled with $1.5 \mathrm{ml} \mathrm{f} / 2$ medium and $0.1 \mathrm{ml}$ of a concentrated algae solution that guaranteed ad libitum food conditions. On day 29, when all individuals were at least 26 days old, we used these virgin worms to assemble different group sizes in order to 
manipulate the level of polyandry. Specifically, we placed virgin worms for $24 \mathrm{~h}$ into groups of two individuals (hereafter called pairs), groups of three individuals (hereafter called triplets) and groups of 16 individuals in 24 well-plates under ad libitum food conditions. For each well-plate we balanced the number of treatments and their positions on the plate.

On day 30 (i.e., 1 day after group formation), we randomly selected one worm out of each group, took morphological measurements and assessed the number of stored sperm in the antrum (see paragraph "Morphological measurement and counts of stored sperm" in the "Methods" section). Next, each worm was isolated in a well of a 24 well-plate. In order to manipulate the food availability, worms were randomly assigned either to wells with a dense algae layer or wells without any algae. Therefore, our manipulation of the food availability consisted of ad libitum food conditions and a complete lack of any food resources after mating. Again, the number of treatments and their positions on the wellplates were balanced among the different well-plates that were used. On days 32, 34, 36, 38 and 44 we transferred all worms to fresh wells, which allowed us to determine the number of the produced offspring on a temporal scale and which prevented the interaction of adult worms with their offspring (embryonic development takes 5 days). After transferring worms to fresh wells we added $0.1 \mathrm{ml}$ of a concentrated algae solution to each old well to guarantee ad libitum food conditions for the developing offspring after hatching. The whole experiment was split into two blocks that were temporally separated by $24 \mathrm{~h}$. Each block initially comprised six replicates for all factor combinations. Blocking had no effect on any of the parameters that we measured ( $t$-tests and Wilcoxon rank sum tests: all $P>0.4)$ and it was therefore ignored in all further analyses.

Female fecundity was defined as the number of offspring produced per day after the isolation of the worms from the group until day 50. Offspring counts were carried out always 10 days after removal of the parental worm from the well, which ensured that all offspring had hatched but that none had matured yet to produce their own offspring.

\section{Rationale for group size manipulation}

In an earlier study we had placed sperm-labelled focal worms together with one, two or 15 unlabelled individuals and these focal worms were allowed to mate for $24 \mathrm{~h}$ under similar conditions as in the current study (e.g., ad libitum food conditions, same enclosure size). We could demonstrate that the number of mating partners of the focal worms was positively affected by the group size. Specifically, the average number of individuals that had received labelled sperm from the focal worm was $0.9,1.5$ and 5.4 in pairs, triplets and groups of 16 individuals, respectively (Janicke and Schärer 2009a). Given that copulations in M. lignano are always reciprocal, the number of individuals that had received labelled sperm should correspond exactly to the number of individuals with which focal individuals copulated in their female sex function. However, the inferred numbers of mating partners probably represent conservative estimates of the level of polyandry since the presence of labelled sperm in the antrum of a worm might underestimate the amount of actually received sperm due to cryptic female choice (Thornhill 1983), sperm displacement (e.g., Waage 1979) or passive sperm loss (e.g., Birkhead and Biggins 1998). Furthermore, one difference in the experimental setup between this study and Janicke and Schärer (2009a) is that we here used virgin worms instead of mated individuals. Given that virgins copulate more frequently than already mated individuals (T. Janicke; unpublished data), we expect that the difference in the level of polyandry between the different group sizes was, if anything, higher in the present study than that reported by Janicke and Schärer (2009a). 
Based on these earlier findings it is very likely that our group size treatment affected the level of polyandry in the present study. However, it is unclear to which extent our manipulation also had an effect on the number of repeated matings with the same individuals.

A number of previous studies on M. lignano showed that these worms are capable of adjusting their sex allocation (i.e., the resource allocation towards the male versus the female function) in response to the group size (e.g., Schärer and Ladurner 2003; Janicke and Schärer 2009b; reviewed in Schärer 2009). In larger groups worms invest more resources into the male function (in terms of larger testes) at a cost to the female function (in terms of smaller ovaries), which reduces the female reproductive output (Schärer et al. 2005; Janicke and Schärer 2009b). However, a recent study indicated that it takes several days of exposure to a specific group size to observe a sex allocation response (Brauer et al. 2007), which suggests that the group size manipulation of only $24 \mathrm{~h}$ in the current study is unlikely to have an effect on the sex allocation of the worms.

\section{Morphological measurement and counts of stored sperm}

We took morphological measurements $24 \mathrm{~h}$ after group formation (directly before isolating worms into single wells) to test whether worms in different treatment groups were similar with regard to their morphology and to examine whether the morphology of a worm correlates with its female fecundity. In particular, we measured body size, testis size and ovary size. We also measured the size of the seminal vesicle, which can be used as an estimate of the number of sperm allocated by worms in previous matings (Schärer and Ladurner 2003). Moreover, we assessed the number of stored sperm in the antrum to test whether this parameter was affected by our group size manipulation and if it was related to female fecundity. On day 50 (20 days after the mating trials) we again counted the number of stored sperm to confirm that all worms had run out of sperm and therefore where unable to produce any further offspring.

All morphological measurements were obtained in vivo in a standardized way (Schärer and Ladurner 2003; Janicke and Schärer 2009b). First, worms were anesthetized by immersing them in a 5:3 mixture of $7.14 \% \mathrm{MgCl}_{2}$ and $\mathrm{f} / 2$ medium for $10 \mathrm{~min}$. Overview pictures of the entire body, the testes, ovaries and the seminal vesicle were taken after compressing worms dorsoventrally to a fixed thickness of $35 \mu \mathrm{m}$ between a microscope slide and a cover slip of a haemocytometer (Schärer and Ladurner 2003). We used a Leica DM 2500 microscope (Leica Microsystems, Germany) to which we connected a digital video camera (DFK 41AF02, The Imaging Source Europe GmbH, Germany) and took digital micrographs at $40 \times$ for body size and $400 \times$ for testis size, ovary size and seminal vesicle size. For image acquisition we used the software BTV Pro 6.0b1 (http://www. bensoftware.com/) and we analysed micrographs using ImageJ 1.42k (http://rsb.info.nih. gov/ij/). All these morphological measurements are repeatable within individuals (Schärer and Ladurner 2003).

Counts of the number of stored sperm in the antrum were carried out directly after morphological measurements using the same optical devices. First, we gently compressed worms between a $24 \times 50 \mathrm{~mm}$ and a $21 \times 26 \mathrm{~mm}$ cover slip using small plasticine feet on each corner of the smaller cover slip as a spacer. Then, we mounted the cover slips with the worm on a modified microscope slide fitted with two raised supports on which the cover slips could be placed. Thereby, the observer could easily flip the compressed worm from the dorsal to the ventral view, which is required to properly count all the sperm that are stored in the antrum. We then recorded QuickTime movies of each antrum at $1,000 \times$ 
magnification (using immersion oil) by focussing two times slowly through the entire organ. Using these movies we later counted the number of stored sperm. All counts were done by the same observer (T.J.) who was blind with regard to the treatment group. In order to assess the repeatability of the sperm counts, the number of stored sperm was assessed twice for all antrum movies except the ones that were recorded 20 days after the mating trials. The analysis of these repeated sperm counts confirmed a high repeatability of the number of stored sperm in the antrum using the method described above (intraclasscorrelation coefficient: $\left.r_{\mathrm{i}}=0.89, F_{36,37}=17.66, P<0.001\right)$.

\section{Statistical analyses}

Initially we aimed to replicate each factor combination 12 times. However, losses during measuring and pipetting errors reduced our final sample size to an average of $9.3 \pm 1.0$ replicates per factor combination (pairs/no food, $N=12$; pairs/ad libitum, $N=8$; triplets/ no food, $N=9$, triplets/ad libitum, $N=6$; groups of 16 individuals/no food, $N=12$; groups of 16 individuals/ad libitum, $N=9$ ).

We tested whether worms exposed to different group sizes were morphologically similar with respect to body size, testis size and ovary size, as intended by our experimental setup. Furthermore, we assessed the effect of group size on the size of the seminal vesicle (our estimate of the amount of sperm allocated during the mating trials) and the number of received sperm. This was done using one-way ANOVAs (or Kruskal-Wallis ANOVAs in case the assumptions for parametric tests were not met). Additionally, we tested whether body size was correlated with the number of stored sperm in order to explore if this trait affected the amount of sperm that an individual is capable of storing or able to obtain from mating partners.

The determinants of female fecundity were assessed using Generalized Linear Mixed Models (GLMMs) with Poisson error distributions and log-link functions (Venables and Ripley 2002) to account for deviations from normality and unequal variances between the treatment groups. In a 'basic model', we included group size, food availability and time since mating as fixed factors, the individual as a random factor and body size and ovary size as covariates. In addition, we fitted an 'extended model', in which we added the number of stored sperm in the antrum (counted $24 \mathrm{~h}$ after group formation) as an additional covariate to the 'basic model'. The reason for running two separate models was that the number of stored sperm could only be assessed from a fraction of all individuals considered in the 'basic model'. This was because many antra contained a ripe egg, which made it impossible to reliably count the number of stored sperm. Therefore, the sample size in the 'extended model' (number of observations $=222$, number of individuals 37 ) was considerably lower compared to the 'basic model' (number of observations $=336$, number of individuals $=56$ ), which means that we presumably had more statistical power to explain variation in female fecundity using the 'basic model'. None of all possible two-way and three-way interaction terms in both the 'basic model' and the 'extended model' explained a significant amount of variation in female fecundity. Therefore, we excluded all interaction terms in the final analyses.

All statistical analyses were carried out in SPSS 17.0 (SPSS Inc. 2008) or R v. 2.10.1 (R Development Core Team 2009). We applied the penalized quasi-likelihood method (PQL) for both GLMMs (Breslow and Clayton 1993) by using the glmmPQL function implemented in the package MASS for R (Venables and Ripley 2010). Values are given as means $\pm 1 \mathrm{SE}$. 


\section{Results}

Worms exposed to different group sizes did not differ significantly in body size, testis size and ovary size when measured directly after the mating trials (one-way ANOVAs: body size, $F_{2,53}=0.02, P=0.977$; testis size, $F_{2,53}=0.52, P=0.600$; ovary size, $F_{2,53}=$ $0.06, P=0.943$; Fig. 1). This suggests that worms in the different groups were initially similar with regard to these morphological traits as intended by our random assignment to the treatment groups. Moreover, this also confirms that the group size manipulation for $24 \mathrm{~h}$ had no significant effect on the sex allocation of the worms. In contrast, seminal

Fig. 1 Comparison of a body size, $\mathbf{b}$ testis size and $\mathbf{c}$ ovary size between individuals exposed for $24 \mathrm{~h}$ to different group sizes, i.e. pairs, triplets and groups of 16 individuals. Bars indicate means \pm 1 SE. Body size, testis size and ovary size do not statistically differ between treatment groups $(P>0.05)$. See text for statistics
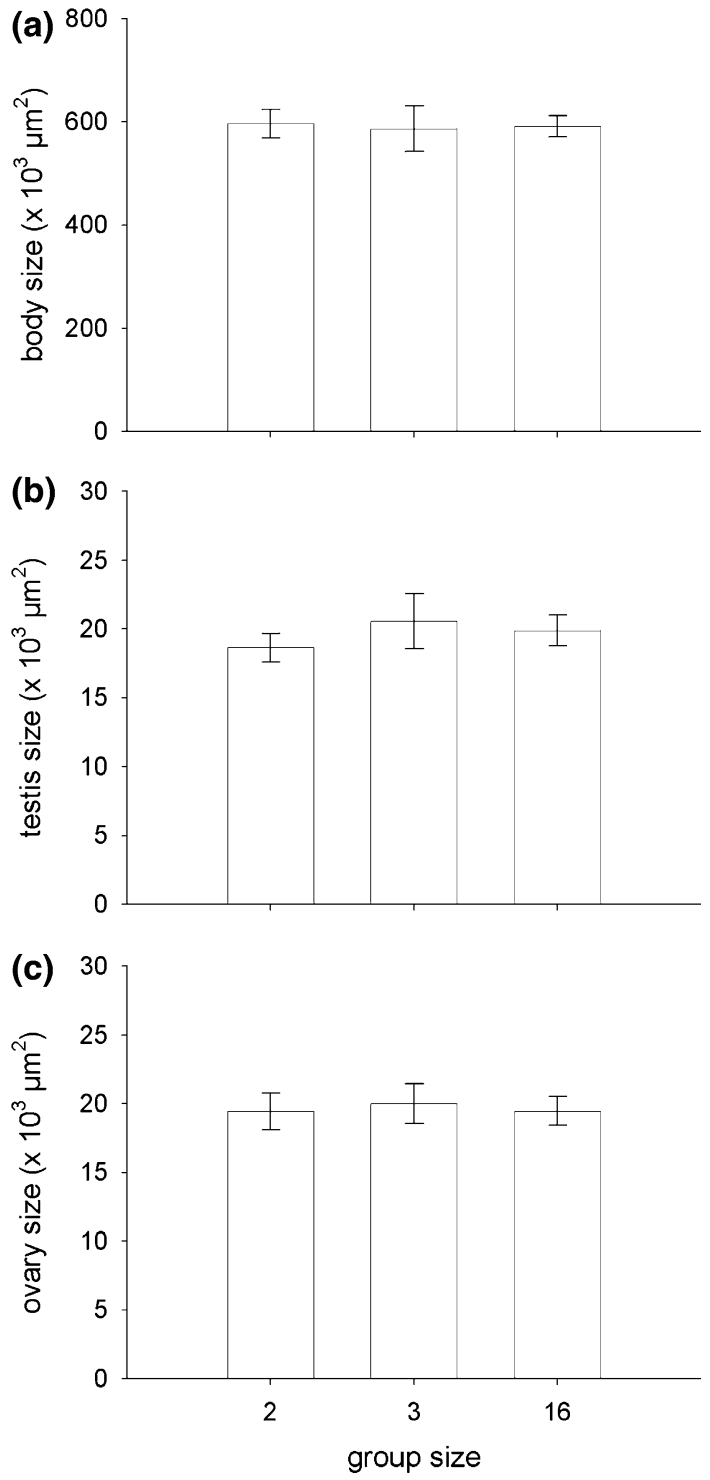
Fig. 2 Comparison of a seminal vesicle size and $\mathbf{b}$ the number of stored sperm in the antrum (i.e., the female sperm-storage organ) between individuals exposed for $24 \mathrm{~h}$ to pairs, triplets and groups of 16 individuals. Boxes show the 25 th percentile, the median and the 75th percentile, whiskers denote the 10th and the 90th percentiles and open circles indicate outliers. Different letters indicate significantly different groups $(P<0.01)$. See text for statistics
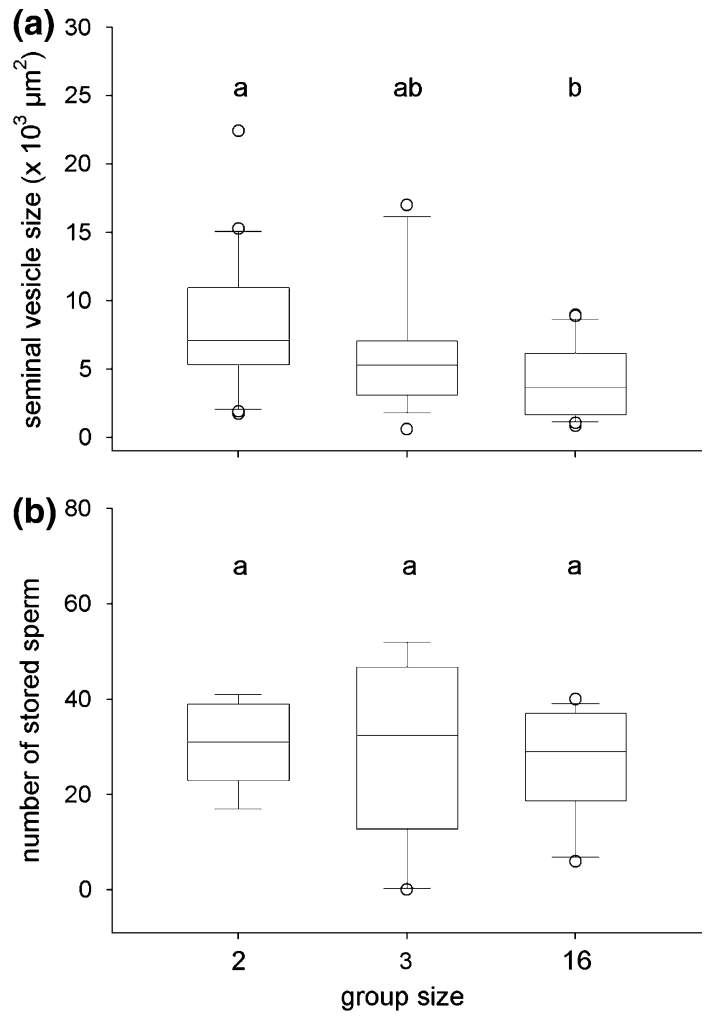

vesicle size was affected by group size (Kruskal-Wallis ANOVA: $\chi^{2}=10.52, d f=2$, $P=0.005$; Fig. 2a). Post hoc comparisons using Mann-Whitney $U$ tests with Bonferroni correction revealed that worms from groups of 16 individuals had smaller seminal vesicles compared to worms from pairs $(z=-3.23, P=0.004, N=41)$, but that there was no statistically significant difference between pairs and triplets $(z=-1.77, P=0.232$, $N=35)$ or between triplets and groups of 16 individuals $(z=-1.14, P=0.764$, $N=36$ ).

Sperm counts conducted directly after the mating trials revealed a high betweenindividual variation in the number of stored sperm in the antrum (mean: $28.6 \pm 2.1$, range: $0-52, N=37$ ). There was only one individual that did not have any sperm in storage. The number of stored sperm did not differ between individuals that were exposed to different group sizes (Kruskal-Wallis ANOVA: $\chi^{2}=1.02, d f=2, P=0.601$; Fig. 2b) and it was not correlated with the body size of the recipient (Pearson correlation coefficient: $r=0.07, P=0.688, N=37$ ). Sperm counts that were carried out 20 days after the mating trials showed that not a single individual still had sperm in storage.

The sum of the offspring produced per worm was on average $5.2 \pm 0.7$ (range: 0-20). In total, there were 11 individuals that did not produce any offspring. Excluding these individuals from the analyses did not qualitatively change the results. We, therefore, report the statistical results from analyses that include these 11 individuals. GLMMs revealed that female fecundity was significantly affected by food availability, the time since mating and the number of stored sperm (Table 1). Specifically, female fecundity was higher in worms 
Table 1 Summary of generalized linear mixed models testing the effect of group size, food availability, time since mating, body size, ovary size and the number of stored sperm on female fecundity

\begin{tabular}{llcrrr}
\hline Model & Source & Estimate $\pm \mathrm{SE}$ & $d f$ & $F$-value & $P$-value \\
\hline Basic model & Group size & - & 2,50 & 0.44 & 0.647 \\
& Food availability & - & 1,50 & 10.14 & 0.003 \\
& Time since mating & - & 5,275 & 26.79 & $<0.001$ \\
& Body size & $0.32 \pm 1.11$ & 1,50 & 0.14 & 0.713 \\
& Ovary size & $-43.29 \pm 27.60$ & 1,50 & 2.46 & 0.123 \\
& Group size & - & 2,30 & 0.13 & 0.880 \\
& Food availability & - & 1,30 & 7.36 & 0.011 \\
& Time since mating & - & 5,180 & 16.80 & $<0.001$ \\
& Body size & $0.49 \pm 1.30$ & 1,30 & 0.24 & 0.627 \\
& Ovary size & $-30.52 \pm 31.94$ & 1,30 & 0.44 & 0.510 \\
& Number of stored sperm & $0.06 \pm 0.02$ & 1,30 & 14.66 & $<0.001$ \\
\hline
\end{tabular}

Given that the number of stored sperm could only be assessed for a fraction of all replicates, we report a 'basic model', which does not include the number of stored sperm and an 'extended model' in which the number of stored sperm was added as a covariate (see paragraph "Statistical analyses" in the "Methods" section)

that were fed ad libitum and that had more sperm in storage after mating (Table 1; Fig. 3a). Moreover, female fecundity decreased rapidly over time (Table 1; Fig. 3a, b). Group size, body size and ovary size had no significant effect on female fecundity (Table 1; Fig. 3b).

\section{Discussion}

The results of our study suggest that it is not the number of mating partners but the food availability that has an effect on female fecundity in the simultaneously hermaphroditic flatworm M. lignano. Worms that were allowed to mate with several different sperm donors produced a similar number of offspring via their female sex function as individuals that could mate with only one sperm donor. In contrast, food resources that were available for egg production had a strong positive effect on female fecundity as predicted by sexual selection theory for simultaneous hermaphrodites (Charnov 1979).

Given that we used only the number of offspring produced under lab conditions as an estimate of female fitness, our results are limited to this measure of hatchling production. It has been argued that non-additive genetic benefits of polyandry are more likely to affect the number of offspring produced rather than offspring quality (Zeh and Zeh 1996; see "Introduction"), and we thus should have been able to detect such genetic benefits. However, there might also have been genetic benefits of polyandry that we were not able to detect in this study. Specifically, genetic benefits derived from mating with many (including overall superior) mating partners might only be apparent when measuring offspring performance, e.g. offspring size, growth rate, or the offspring's own reproductive success (e.g., Ojanguren et al. 2005; Fisher et al. 2006).

In this study we manipulated the level of polyandry by exposing worms to different group sizes, which has been previously shown to affect the number of mating partners in $M$. lignano. Based on these earlier findings, we expect that the average number of mating partners in pairs, triplets and groups of 16 individuals was at least 0.9, 1.5 and 5.4 individuals, respectively (Janicke and Schärer 2009a; see also paragraph on the "Rationale for 

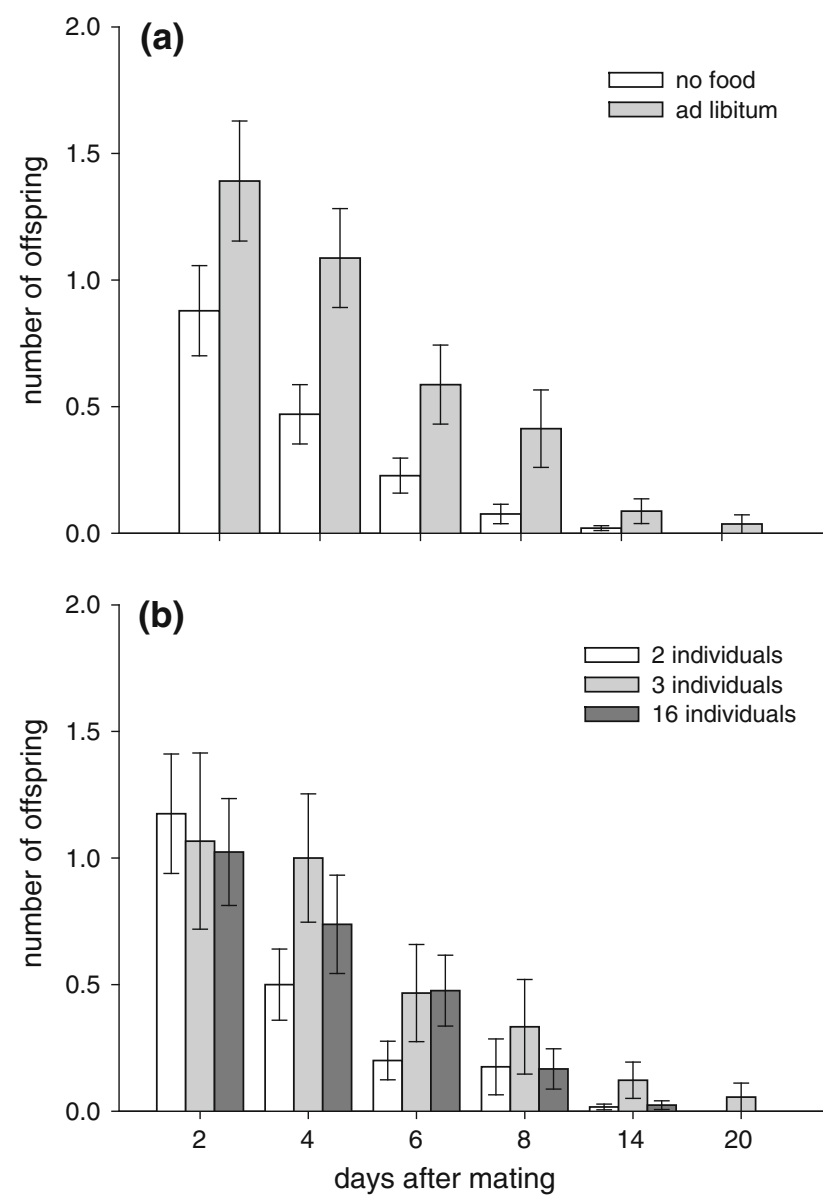

Fig. 3 Daily female fecundity after mating shown for $\mathbf{a}$ the feeding levels and $\mathbf{b}$ the different group sizes. Data shown are lumped across group sizes in panel $\mathbf{a}$ and across feeding levels in panel $\mathbf{b}$. Note that $x$-axes are not linearly scaled. Bars indicate means $\pm 1 \mathrm{SE}$. See text for statistics

group size manipulation" in the "Methods" section). Therefore, we are confident that we managed to manipulate the level of polyandry substantially in the current study. Nevertheless, the group size manipulation might not only have influenced the number of mating partners, but also the number of repeated matings with the same partners, for which we were unable to control with our experimental setup. Therefore, we can not exclude that our manipulation of polyandry was confounded by an additional effect of group size on the number of matings. Until now, we have no data on the effect of group size on the mating rate for the particular situation in which worms were kept in our experiment. Previous mating experiments, which were conducted in so-called observation chambers (in which worms are allowed to copulate in very small drops of culture medium; for details see Schärer et al. 2004), revealed no difference in the per capita mating rate between groups of 2, 3 and 4 individuals (T. Janicke, unpublished data). On the one hand, this previous study shows that repeated matings with the same mating partner clearly do occur in small groups. On the other hand, the results suggest that the group size has no direct effect on the mating 
rate in $M$. lignano. However, we have to clarify that in the current study all mating trials were carried out in much larger enclosures and the maximum number of worms was considerably higher, which limits the comparability of both studies.

In the current study we found that the size of the seminal vesicle was smaller in worms that were exposed to larger groups, which means that worms spent more sperm when they had the opportunity to mate with more mating partners. The most parsimonious explanation for this effect is a higher mating rate in larger groups. However, the observed effect could also indicate that worms allocated more sperm per mating in more competitive situations, as predicted by sperm competition theory (e.g., Parker 1998). Consequently, it is not possible to infer from our data, whether the group size manipulation had an effect on the mating rate and therefore on the number of repeated matings. Irrespective of possible effects on the frequency of repeated matings, we are certain that our experimental setup induced variation in the level of polyandry between the different group sizes. On the assumption that the mating rate was constant across all groups, our data indicate that polyandry has no effect on female fecundity in M. lignano. If the worms mated more frequently in larger groups, our results suggest that both polyandry and repeated mating have no positive or negative effect on our measure of female reproductive output. Only if polyandry and repeated matings have opposing fitness consequences for the female sex function (e.g., mating with different partners is beneficial but mating several times with the same partner incurs fitness costs) and if the per capita mating rate differed between groups, we might have been unable to detect an effect of polyandry on the female fecundity with our experimental setup.

Studies on the fitness consequences of multiple mating for the female sex function in simultaneously hermaphroditic animals have primarily focused on the role of repeated matings rather than the level of polyandry. For instance, in the land snail Arianta arbustorum repeated matings in the female role lead to an increased number of eggs laid, but not to a difference in the number of hatchlings produced (Chen and Baur 1993). In contrast, in the hermaphroditic freshwater snail Lymnea stagnalis it has been shown that individuals that were allowed to mate in groups lay fewer eggs than isolated individuals and an experimental manipulation of the number of copulations indicated that this difference was due to costs associated with mating (van Duivenboden et al. 1985; but see Koene et al. 2006). Recently, it has been demonstrated that at least a part of the negative effect of mating rate on female fecundity in L. stagnalis is due to the receipt of seminal fluids containing male accessory gland products, which presumably suppresses egg laying (Koene et al. 2009). Although these studies on L. stagnalis did not explicitly differentiate between effects of polyandry and repeated matings, they suggest that mating with the same partner can be costly for the female sex function in this simultaneously hermaphroditic snail.

Probably the most conclusive study on the influence of polyandry on female reproduction has been carried out in a simultaneously hermaphroditic opisthobranch Chelidonura sandrana (Sprenger et al. 2008). Similar to our findings, polyandry had no effect on the total number of egg masses produced and the proportion of fertile eggs. However, egg capsule volume and larval length was higher in individuals that mated once with four different sperm donors compared to individuals that mated four times with the same sperm donor, suggesting that the level of polyandry affected maternal provisioning (Sprenger et al. 2008). A positive effect of polyandry on female fecundity has also been reported for the broadcast spawning hermaphroditic ascidian Pyura stolonifera, in which a mixture of ejaculates from different donors increased the hatching success as a result of an 
elevated fertilization success compared to ejaculates from single sperm donors (Marshall and Evans 2007).

The positive effect of food availability on female fecundity found in our study is not very surprising, since resources available for egg production are expected to be crucial for the reproductive output of the female sex function (Charnov 1979). More surprising is that the initial body size and ovary size did not significantly predict the female reproductive output. This might either mean that both traits are indeed not correlated with female fecundity or that the variation in both morphological traits was too small to find an effect. The latter explanation is supported by a previous study in $M$. lignano, which suggests that if one induces variation in ovary size experimentally, a positive effect on the female reproductive output becomes apparent (Schärer et al. 2005).

The only covariate that was correlated with female fecundity was the number of stored sperm. Individuals that managed to store more sperm produced more offspring. However, whether this effect is relevant under more natural conditions is questionable, since fecundity was assessed in isolated worms, which could not replenish their sperm reserves after the mating trials. Given that the mating rate in M. lignano is relatively high (Schärer et al. 2004) and that worms can occur at relatively high densities in the field (K. Sekii et al., unpublished data) it remains unclear whether access to received sperm can constrain female fecundity under natural conditions, a question that should be studied in the field.

Remarkably, the number of stored sperm was unaffected by the group size. Therefore, the female sex function does not seem to gain direct benefits from multiple mating in terms of replenishing the own sperm reserves. Instead, variation in the number of stored sperm was presumably induced by factors that are not linked to multiple mating. For instance, the size of the female sperm-storage organ might constrain the amount of sperm an individual is capable of storing and may thereby affect the number of offspring produced. Moreover, quality traits of the sperm recipient might have an effect on the number of sperm that sperm donors transfer during copulations as predicted by theoretical models on strategic sperm allocation (e.g., Reinhold et al. 2002). So far, very little is known about how intrinsic traits of recipients influence the number of stored sperm in M. lignano. In this study, we found no correlation between the body size and the number of stored sperm, which suggests that body size itself and other traits that are size-dependent do not affect the number of sperm an individual is able to store or obtain from its mating partners.

Although the number of stored sperm was unaffected by group size, individuals in larger groups allocated more sperm during the mating trials (as inferred from the size of the seminal vesicle). This clearly suggests that not all sperm that are transferred during mating are finally stored in the partner. First of all, this could simply be due to passive sperm loss during egg laying, because fertilized eggs have to pass through the antrum before they are laid. Similarly, the capacity of the antrum is finite and therefore some of the transferred sperm may never become stored and therefore get lost passively. Another potential explanation is sperm displacement, in which individuals actively displace some of the stored sperm from previous mates. Alternatively, the recipient itself may remove the sperm out of its own antrum in order to digest them or to bias paternity towards favoured mating partners. Indeed, after copulating, worms often bend themselves in order to touch their female genital opening with their pharynx and then appear to suck sperm out of the antrum (Schärer et al. 2004). So far we know very little about sperm displacement and cryptic female choice in M. lignano (for a description of morphologies and behaviours that might facilitate both processes see Vizoso et al. 2010).

To summarize, in our study food availability but not group size (used as a proxy for the level of polyandry) had an effect on female fecundity in the hermaphroditic flatworm 
M. lignano. This finding is consistent with classical sexual selection theory (Bateman 1948), which predicts that the female reproductive output primarily depends on the resources that are available for egg production rather than on the number of mates. Therefore, our results support the hypothesis formulated by Charnov (1979), that Bateman's principle can also be applied to simultaneously hermaphroditic animals. However, in order to provide an ultimate test of Bateman's principle for M. lignano, one needs to assess the fitness benefits of multiple mating for both the female and the male sex function.

Acknowledgments We thank Dita B. Vizoso for technical assistance. Ralph Dobler and two anonymous referees provided constructive comments on an earlier version of the manuscript. We also thank Lucas Marie-Orléach and Matthew D. Hall for statistical advice. Finally, we are grateful to Jürgen Hottinger, Viktor Mislin and Urs Stiefel for technical support. This study was funded by grants from the Swiss National Science Foundation to L.S. (3100A0-113708 and 3100A0-127503).

\section{References}

Andersen RA, Berges JA, Harrison PJ, Watanabe MM (2005) Recipes for freshwater and seawater media. In: Andersen RA (ed) Algal culturing techniques. Elsevier, Amsterdam, pp 429-538

Andersson M (2005) Evolution of classical polyandry: three steps to female emancipation. Ethology 111: $1-23$

Anthes N, Putz A, Michiels NK (2005) Gender trading in a hermaphrodite. Curr Biol 15:R792-R793

Arnqvist G, Nilsson T (2000) The evolution of polyandry: multiple mating and female fitness in insects. Anim Behav 60:145-164

Baker RH, Ashwell RIS, Richards TA, Fowler K, Chapman T, Pomiankowski A (2001) Effects of multiple mating and male eye span on female reproductive output in the stalk-eyed fly, Cyrtodiopsis dalmanni. Behav Ecol 12:732-739

Bateman AJ (1948) Intra-sexual selection in Drosophila. Heredity 2:349-368

Bedhomme S, Bernasconi G, Koene JM, Lankinen A, Arathi HS, Michiels NK, Anthes N (2009) How does breeding system variation modulate sexual antagonism? Biol Lett 5:717-720

Birkhead TR, Biggins JD (1998) Sperm competition mechanisms in birds: models and data. Behav Ecol 9:253-260

Brauer VS, Schärer L, Michiels NK (2007) Phenotypically flexible sex allocation in a simultaneous hermaphrodite. Evolution 61:216-222

Breslow NE, Clayton DG (1993) Approximate inference in generalized linear mixed models. J Am Stat Assoc 88:9-25

Bybee LF, Millar JG, Paine TD, Campbell K, Hanlon CC (2005) Effects of single versus multiple mates: monogamy results in increased fecundity for the beetle Phoracantha semipunctata. J Insect Behav 18:513-527

Chapman T, Liddle LF, Kalb JM, Wolfner MF, Partridge L (1995) Cost of mating in Drosophila melanogaster females is mediated by male accessory-gland products. Nature 373:241-244

Charnov EL (1979) Simultaneous hermaphroditism and sexual selection. Proc Natl Acad Sci USA 76: $2480-2484$

Chen XF, Baur B (1993) The effect of multiple mating on female reproductive success in the simultaneously hermaphroditic land snail Arianta arbustorum. Can J Zool 71:2431-2436

Crudgington HS, Siva-Jothy MT (2000) Genital damage, kicking and early death-the battle of the sexes takes a sinister turn in the bean weevil. Nature 407:855-856

Daly M (1978) Cost of mating. Am Nat 112:771-774

Diaz SA, Haydon DT, Lindstrom J (2010) Sperm-limited fecundity and polyandry-induced mortality in female nematodes Caenorhabditis remanei. Biol J Linn Soc 99:362-369

Dunn DW, Sumner JP, Goulson D (2005) The benefits of multiple mating to female seaweed flies, Coelopa frigida (Diptera: Coelpidae). Behav Ecol Sociobiol 58:128-135

Engqvist L (2007) Nuptial food gifts influence female egg production in the scorpionfly Panorpa cognata. Ecol Entomol 32:327-332

Evans JP, Magurran AE (2000) Multiple benefits of multiple mating in guppies. Proc Natl Acad Sci USA 97:10074-10076 
Evans JP, Marshall DJ (2005) Male-by-female interactions influence fertilization success and mediate the benefits of polyandry in the sea urchin Heliocidaris erythrogramma. Evolution 59:106-112

Fedorka KM, Mousseau TA (2002) Material and genetic benefits of female multiple mating and polyandry. Anim Behav 64:361-367

Fischer EA (1980) The relationship between mating system and simultaneous hermaphroditism in the coralreef fish, Hypoplectrus nigricans (Serranidae). Anim Behav 28:620-633

Fisher DO, Double MC, Moore BD (2006) Number of mates and timing of mating affect offspring growth in the small marsupial Antechinus agilis. Anim Behav 71:289-297

Fjerdingstad EJ, Boomsma JJ (1998) Multiple mating increases the sperm stores of Atta colombica leafcutter ant queens. Behav Ecol Sociobiol 42:257-261

Green K, Tregenza T (2009) The influence of male ejaculates on female mate search behaviour, oviposition and longevity in crickets. Anim Behav 77:887-892

Gwynne DT (1984) Courtship feeding increases female reproductive success in bush-crickets. Nature 307:361-363

Hosken DJ, Stockley P (2003) Benefits of polyandry: a life history perspective. Evol Biol 33:173-194

House CM, Walling CA, Stamper CE, Moore AJ (2009) Females benefit from multiple mating but not multiple mates in the burying beetle Nicrophorus vespilloides. J Evol Biol 22:1961-1966

Janicke T, Schärer L (2009a) Determinants of mating and sperm-transfer success in a simultaneous hermaphrodite. J Evol Biol 22:405-415

Janicke T, Schärer L (2009b) Sex allocation predicts mating rate in a simultaneous hermaphrodite. Proc R Soc B-Biol Sci 276:4247-4253

Jennions MD, Petrie M (2000) Why do females mate multiply? A review of the genetic benefits. Biol Rev 75:21-64

Klemme I, Eccard JA, Ylonen H (2007) Why do female bank voles, Clethrionomys glareolus, mate multiply? Anim Behav 73:623-628

Koene JM, Montagne-Wajer K, Ter Maat A (2006) Effects of frequent mating on sex allocation in the simultaneously hermaphroditic great pond snail (Lymnaea stagnalis). Behav Ecol Sociobiol 60: $332-338$

Koene JM, Brouwer A, Hoffer JNA (2009) Reduced egg laying caused by a male accessory gland product opens the possibility for sexual conflict in a simultaneous hermaphrodite. Anim Biol 59:435-448

LaDage LD, Gutzke WHN, Simmons RA, Ferkin MH (2008) Multiple mating increases fecundity fertility and relative clutch mass in the female leopard gecko (Eublepharis macularius). Ethology 114:512-520

Ladurner P, Schärer L, Salvenmoser W, Rieger RM (2005) A new model organism among the lower Bilateria and the use of digital microscopy in taxonomy of meiobenthic Platyhelminthes: Macrostomum lignano, n. sp. (Rhabditophora, Macrostomorpha). J Zool Syst Evol Res 43:114-126

Leonard JL (2005) Bateman's principle and simultaneous hermaphrodites: a paradox. Integr Comp Biol 45:856-873

Leonard JL (2006) Sexual selection: lessons from hermaphrodite mating systems. Integr Comp Biol 46: 349-367

Leonard JL, Lukowiak K (1991) Sex and the simultaneous hermaphrodite-testing models of male-female conflict in a sea slug, Navanax inermis (Opisthobranchia). Anim Behav 41:255-266

Levitan DR, Petersen C (1995) Sperm limitation in the sea. Trends Ecol Evol 10:228-231

Marshall DJ, Evans JP (2007) Context-dependent genetic benefits of polyandry in a marine hermaphrodite. Biol Lett 3:685-688

Michiels NK (1998) Mating conflicts and sperm competition in simultaneous hermaphrodites. In: Birkhead T, Møller AP (eds) Sperm competition and sexual selection. Academic Press, London, pp 219-254

Michiels NK, Koene JM (2006) Sexual selection favors harmful mating in hermaphrodites more than in gonochorists. Integr Comp Biol 46:473-480

Ojanguren AF, Evans JP, Magurran AE (2005) Multiple mating influences offspring size in guppies. J Fish Biol 67:1184-1188

Orsetti DM, Rutowski RL (2003) No material benefits, and a fertilization cost, for multiple mating by female leaf beetles. Anim Behav 66:477-484

Parker GA (1998) Sperm competition and the evolution of ejaculates: towards a theory base. In: Birkhead T, Møller AP (eds) Sperm competition and sexual selection. Academic Press, Cambridge, pp 3-54

Pitnick S (1993) Operational sex-ratios and sperm limitation in populations of Drosophila pachea. Behav Ecol Sociobiol 33:383-391

Priest NK, Roach DA, Galloway LF (2008) Cross-generational fitness benefits of mating and male seminal fluid. Biol Lett 4:6-8

R Development Core Team (2009) R: a language and environment for statistical computing, 2.10.1. $\mathrm{R}$ Foundation for Statistical Computing, Vienna 
Reinhardt K, Naylor RA, Siva-Jothy MT (2009) Ejaculate components delay reproductive senescence while elevating female reproductive rate in an insect. Proc Natl Acad Sci USA 106:21743-21747

Reinhold K, Kurtz J, Engqvist L (2002) Cryptic male choice: sperm allocation strategies when female quality varies. J Evol Biol 15:201-209

Reynolds JD (1996) Animal breeding systems. Trends Ecol Evol 11:A68-A72

Rolff J, Siva-Jothy MT (2002) Copulation corrupts immunity: a mechanism for a cost of mating in insects. Proc Natl Acad Sci USA 99:9916-9918

Ronkainen K, Kaitala A, Kivelä SM (2010) Polyandry, multiple mating, and female fitness in a water strider Aquarius paludum. Behav Ecol Sociobiol 64:657-664

Rowe L (1994) The costs of mating and mate choice in water striders. Anim Behav 48:1049-1056

Schärer L (2009) Tests of sex allocation theory in simultaneously hermaphroditic animals. Evolution 63:1377-1405

Schärer L, Ladurner P (2003) Phenotypically plastic adjustment of sex allocation in a simultaneous hermaphrodite. Proc R Soc B-Biol Sci 270:935-941

Schärer L, Joss G, Sandner P (2004) Mating behaviour of the marine turbellarian Macrostomum sp.: these worms suck. Mar Biol 145:373-380

Schärer L, Sandner P, Michiels NK (2005) Trade-off between male and female allocation in the simultaneously hermaphroditic flatworm Macrostomum sp. J Evol Biol 18:396-404

Schwartz SK, Peterson MA (2006) Strong material benefits and no longevity costs of multiple mating in an extremely polyandrous leaf beetle, Chrysochus cobaltinus (Coleoptera: Chrysomelidae). Behav Ecol 17:1004-1010

Sella G (1985) Reciprocal egg trading and brood care in a hermaphroditic polychaete worm. Anim Behav 33:938-944

Simmons LW (2005) The evolution of polyandry: sperm competition, sperm selection, and offspring viability. Annu Rev Ecol Evol Syst 36:125-146

Sprenger D, Anthes N, Michiels NK (2008) Multiple mating affects offspring size in the opisthobranch Chelidonura sandrana. Mar Biol 153:891-897

SPSS Inc. (2008) SPSS for windows, version 17.0. SPSS Inc, Chicago

Thornhill R (1983) Cryptic female choice and its implications in the scorpionfly Harpobittacus nigriceps. Am Nat 122:765-788

Thrall PH, Antonovics J, Bever JD (1997) Sexual transmission of disease and host mating systems: within-season reproductive success. Am Nat 149:485-506

Tregenza T, Wedell N (1998) Benefits of multiple mates in the cricket Gryllus bimaculatus. Evolution $52: 1726-1730$

Vahed K (1998) The function of nuptial feeding in insects: review of empirical studies. Biol Rev 73:43-78

van Duivenboden YA, Pieneman AW, Termaat A (1985) Multiple mating suppresses fecundity in the hermaphrodite freshwater snail Lymnaea stagnalis - a laboratory study. Anim Behav 33:1184-1191

Venables WN, Ripley BD (2002) Modern applied statistics with S, 4th edn. Springer, New York

Venables WN, Ripley BD (2010) MASS: main package of Venables and Ripley's MASS, R package, version $7.3-5$

Vizoso DB, Rieger G, Schärer L (2010) Goings-on inside a worm: functional hypotheses derived from sexual conflict thinking. Biol J Linn Soc 99:370-383

Vreys C, Michiels NK (1998) Sperm trading by volume in a hermaphroditic flatworm with mutual penis intromission. Anim Behav 56:777-785

Waage JK (1979) Dual function of the damselfly penis—-sperm removal and transfer. Science 203:916-918

Wagner WE, Kelley RJ, Tucker KR, Harper CJ (2001) Females receive a life-span benefit from male ejaculates in a field cricket. Evolution 55:994-1001

Watson PJ (1991) Multiple paternity as genetic bet-hedging in female Sierra Dome Spiders, Linyphia litigiosa (Linyphiidae). Anim Behav 41:343-360

Yasui Y (1997) A “'good-sperm' model can explain the evolution of costly multiple mating by females. Am Nat 149:573-584

Yasui Y (1998) The 'genetic benefits' of female multiple mating reconsidered. Trends Ecol Evol 13: 246-250

Zeh JA, Zeh DW (1996) The evolution of polyandry I: intragenomic conflict and genetic incompatibility. Proc R Soc B-Biol Sci 263:1711-1717

Zeh JA, Zeh DW (1997) The evolution of polyandry II: post-copulatory defences against genetic incompatibility. Proc R Soc B-Biol Sci 264:69-75

Zeh JA, Zeh DW (2001) Reproductive mode and the genetic benefits of polyandry. Anim Behav 61: $1051-1063$ 